\title{
Pelvic and perineal complications of Crohn's disease: assessment using magnetic resonance imaging
}

P J Haggett, N R Moore, J D Shearman, S P L Travis, D P Jewell, N J Mortensen

\begin{abstract}
This study evaluated the role of magnetic resonance imaging (MRI) in the demonstration of the pelvic and perianal complications of Crohn's disease. Twenty five patients with active Crohn's disease were studied (12 male; mean age $41 \cdot 1$ years). MRI examinations were performed using a 1.5 Tesla system, within 14 days after clinical assessment. $T 1$ and $T 2$ weighted fast spin echo sequences in two or three orthogonal planes were performed, with fat suppression in some cases. The MRI results were correlated with surgical and clinical findings. In 16 patients, cutaneous, deep perineal or enterovesical fistulas or abscesses were diagnosed at MRI which showed close correlation with findings at examination under anaesthetic. In eight patients no fistulas or abscesses were seen at MRI nor was there any evidence of complications on clinical examination and flexible sigmoidoscopy. There was one false negative examination in a patient who had a colovesical fistula. In conclusion, MRI can accurately show the pelvic and perineal complications of Crohn's disease and may render examination under anaesthetic unnecessary.

(Gut 1995; 36: 407-410)
\end{abstract}

Keywords: Crohn's disease, magnetic resonance imaging.

John Radcliffe Hospital and University of Oxford, Oxford

Correspondence to: Dr P J Haggett, Departmen of Radiology, John Radcliffe Hospital, Headington, Oxford OX3 9DU.

Accepted for publication 9 June 1994

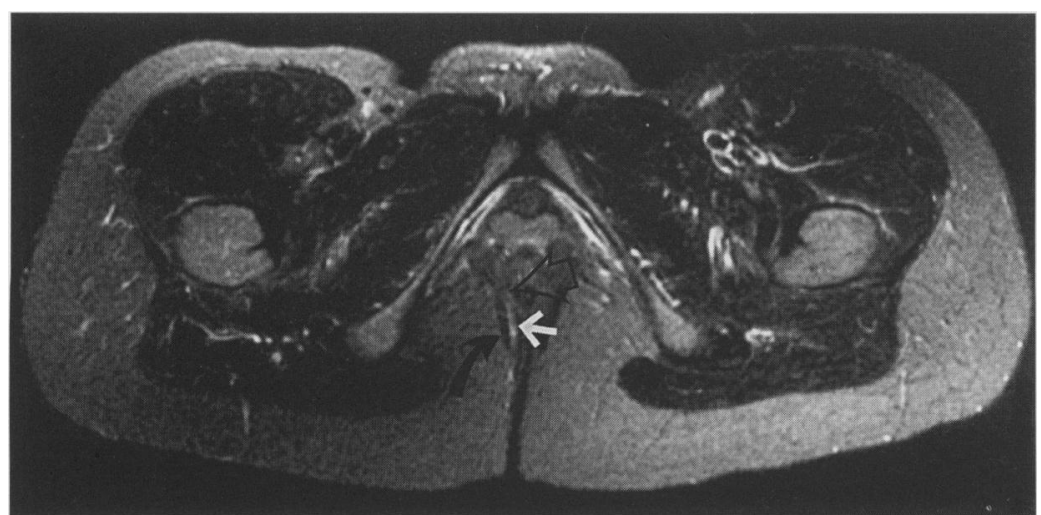

Figure 1: Transverse T2 weighted image (repetition time $(T R)=4000 \mathrm{~ms}$, effective echo time $(T E)=102 \mathrm{~ms}$ ) with suppression of the fat signal, at the level of the external anal sphincter. The high signal track (arrow) is a simple trans-sphincteric fistula, passing between the internal sphincter (open arrow) and the puborectalis (curved arrow). inflammation and fistula in ano is particularly common in patients with Crohn's disease. The nature and extent of these complications determine the choice of medical or surgical treatment. ${ }^{2}$ We have performed a prospective study to determine the value of magnetic resonance imaging (MRI) in assessing the pelvic and perineal complications of Crohn's disease.

\section{Methods}

We examined 25 patients (mean age 41.4 years, 12 male). All patients had symptoms or signs of active pelvic Crohn's disease; perineal pain, anal discharge, rectal bleeding or pneumaturia. All patients had a clinical examination and flexible sigmoidoscopy carried out by a consultant or senior registrar in gastroenterology, or examination under anaesthetic performed by a consultant or senior registrar in colorectal surgery. The clinical assessment was performed before MRI in seven patients and after MRI in 18 patients.

The MRI examinations were performed on a high field machine operating at 1.5 Tesla (International General Electric Medical Systems, Slough) within 14 days of clinical assessment. All patients had a T1 weighted fast spin echo acquisition performed in the coronal plane (repetition time (TR) $=700 \mathrm{~ms}$, effective echo time $(\mathrm{TE})=17 \mathrm{~ms}$, echotrain=4). Subsequently, axial and sagittal or coronal T2 weighted fast spin echo sequences were acquired ( $T R=4000 \mathrm{~ms}, \mathrm{TE}=102 \mathrm{~ms}$, echotrain $=16$ ). The body transmit and receive coil was used in all examinations. The acquisition matrix was either 512 by 256 or 512 by 512 , and the field of view was $40-48 \mathrm{~cm}$. The in plane resolution varied from $0.8 \mathrm{~mm} \times 0.8 \mathrm{~mm}$ to $0.9 \mathrm{~mm} \times 1.8 \mathrm{~mm}$. In the early part of the study, T1 weighted images were also performed in the axial or sagittal plane. Most of the T2 weighted sequences were performed with suppression of the fat signal using the frequency specific spectral presaturation technique.

Both the MRI examinations and the clinical assessment were carried out without prior knowledge of the other worker's findings.

The results of the MRI examinations and the clinical assessments were then compared.

\section{Results}

Sixteen of 25 patients had perianal fistulas or pelvic abscesses diagnosed at examination under anaesthetic. There was accurate correlation of the MRI appearances with the findings 


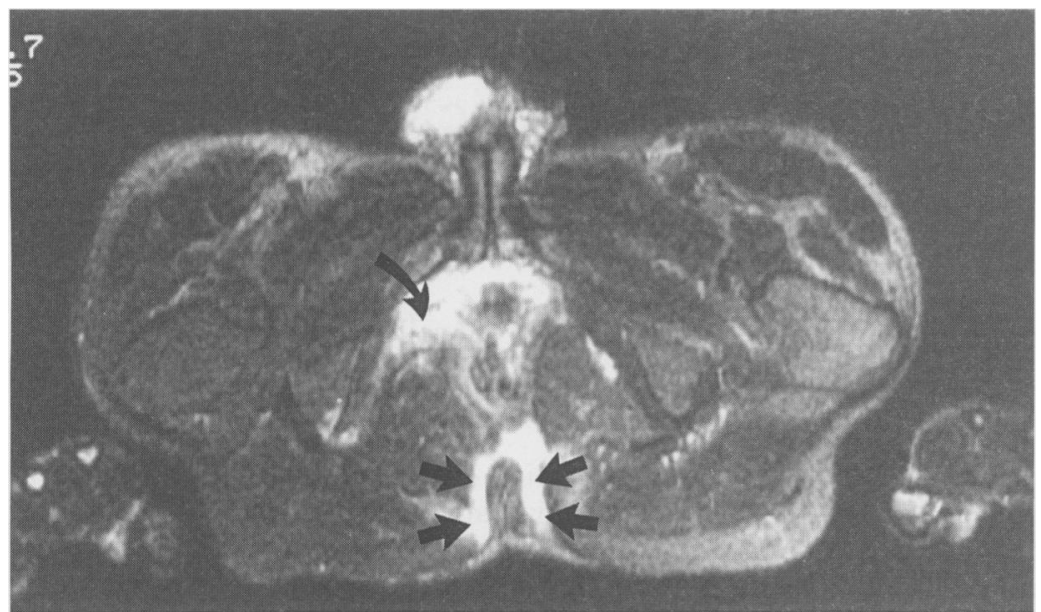

Figure 2: Transverse $T 2$ weighted image $(T R=4000 \mathrm{~ms}, T E=102 \mathrm{~ms})$ with fat suppression, showing a complex fistula on both sides of the natal cleft (arrows) with extensive inflammation affecting the right obturator internus muscle (curved arrow).

at examination under anaesthetic. A single fistula was diagnosed in six patients (Fig 1). Multiple abscesses and fistulas were seen in 10 patients (Fig 2). MRI showed additional fistulas or abscesses in three patients and within this group, there were two patients in whom MRI showed deep and unsuspected pararectal or ischiorectal abscesses (Figs 3 and 4).

Figure 3: (A) Coronal T2 weighted image $(T R=4000$ $\mathrm{ms}, T E=102 \mathrm{~ms}$ ) with fat suppression, showing bilateral fluid collections (multiloculated on the left) in the ischiorectal fossae (arrows). (B) Transverse $T 2$ weighted $(T R=4000$ $m s, T E=102 \mathrm{~ms}$ ) with fat suppression. This confirms the ischiorectal high signal fluid collections (arrows). The low signal focus on the right (curved arrow) is either gas or an area of calcification. No deep abscess formation was suspected on clinical examination or at examination under anaesthetic.
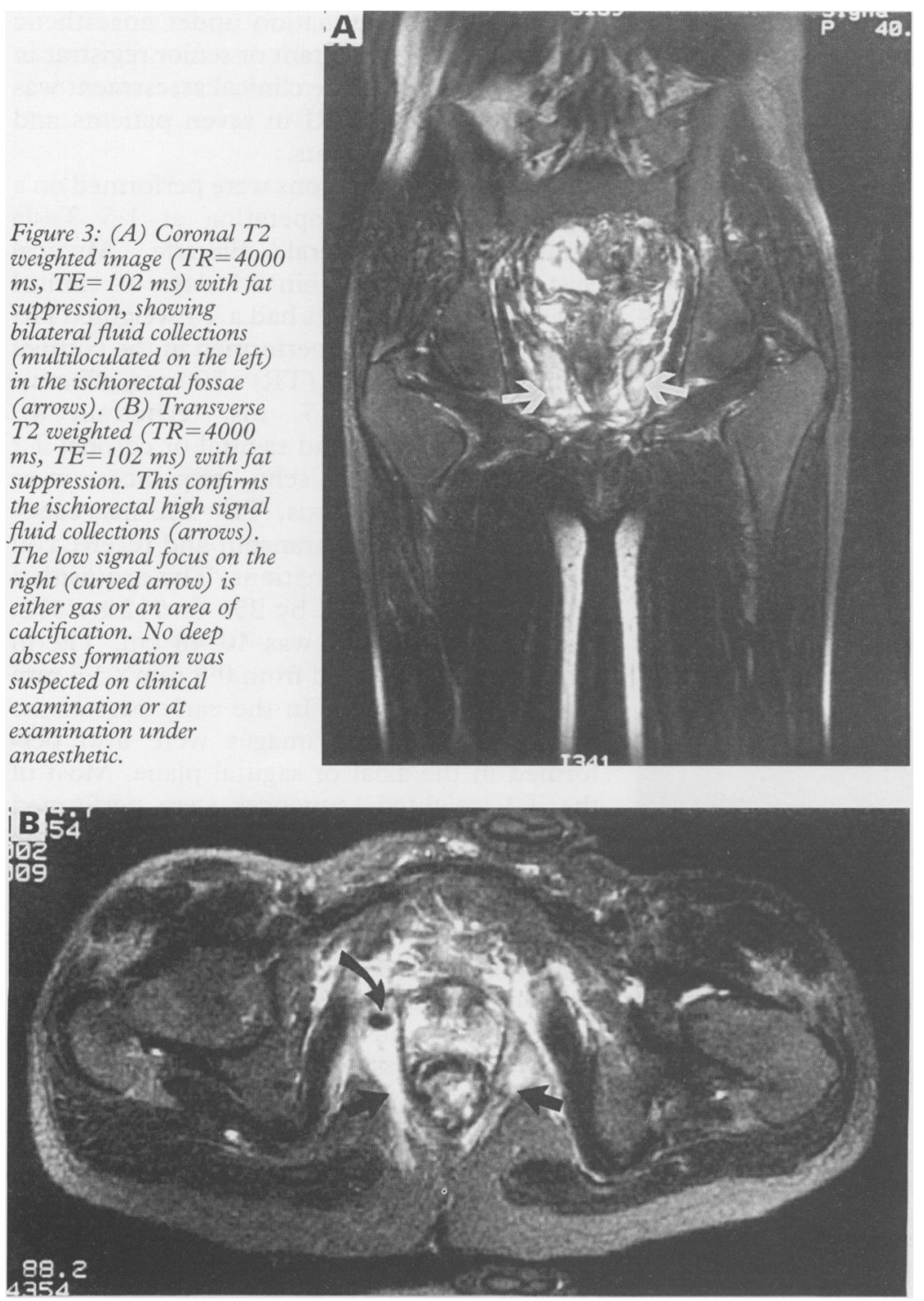

In eight of 25 patients, no pelvic abscess or fistula was diagnosed at MRI. Clinical assessment in all of these patients did not show any complication.

In one patient in whom a pelvic abscess was suspected, the MRI examination was initially thought to be normal. On review, however, a small loculus of gas in the bladder with bladder dome thickening was identified on one axial section, and an adjacent colonic loop was seen. At subsequent laparotomy, a colovesical fistula was found.

\section{Discussion}

Perianal infection is thought to originate within the anal glands. The presence of lymphoid tissue around these glands may account for the increased incidence of perianal involvement in Crohn's disease. ${ }^{3}$ The infection may extend as a fistula in the plane between the internal and external anal sphincters (intersphincteric), or may penetrate the external sphincter (transphincteric). More complicated fistulas may extend around the rectum as a horseshoe abscess, or perforate through the pelvic floor (suprasphincteric). In some patients, particularly those with Crohn's disease, the inflammation may extend from the rectum to the perianal region and not affect the sphincter mechanism (extrasphincteric). ${ }^{2}$

Careful digital examination under anaesthetic is regarded as the ideal standard for the diagnosis of fistula in ano. Even experienced surgeons, however, may fail to detect the internal opening in up to $13 \%$ of cases. ${ }^{4}$ In addition to bidigital palpation, probing of fistula tracks is commonly performed. Considerable care must be taken because injudicious probing may transform a transphincteric fistula into an extrasphincteric fistula, with grave implications to the patient. ${ }^{2}$ Accurate assessment of the anatomy is vital because an incorrect diagnosis may lead to inappropriate surgery. Division of the internal and external sphincters will result in loss of continence.

The need for accurate imaging is evident. Fistulography will delineate a fistula track if the external orifice can be cannulated, but it gives little information about immediate anatomical relations. ${ }^{5}$ Fistulography may also fail to show the complete extent of complex fistulas and deep abscesses. Computed tomography is successful in showing most perianal fistulas and abscesses but requires the use of oral, rectal, and intravenous contrast media. Some fistulas escape detection, or may be diagnosed as inflammatory streaking only, if air or barium do not enter the track. ${ }^{6}$ The substantial radiation dose to the pelvis in this group of young patients is undesirable. ${ }^{7}$

Anal endosonography has many advantages when used by experienced operators and may detect simple fistulas with an accuracy equal to that of examination under anaesthetic. ${ }^{4}$ Endosonography is not reliable, however, in the detection of extrasphincteric and suprasphincteric fistulas. The short focal length prohibits the detection of ischiorectal abscesses. ${ }^{4}$ 
Endosonography can be painful and difficulties occur with previous sepsis or surgery because endosonography cannot distinguish the low reflectivity track of a fistula from that of scar tissue.

MRI has many advantages in the assessment of the pelvis. It offers excellent soft tissue discrimination, multiplanar image acquisition, a wide field of view, and it does not use ionising radiation. The coronal acquisition plane is particularly valuable in the assessment of perianal disease because the relation of a fistula in ano to the levator ani and puborectalis muscles (Fig 4) is crucial for surgical planning.

In this study, MRI correctly identified surgically verified perianal fistulas in all patients. MRI correctly classified six of six simple fistulas and showed multiple fistulas in 10 of 10 . Furthermore, MRI showed additional complications in three patients, and in two patients MRI showed deep and unsuspected abscesses (Figs 3 and 4). Rounded areas of high signal on T2 images were diagnosed as abscesses. Linear high signal may represent fluid or inflammation in fistula tracks. Enhancement of signal on T1 images after gadolinium diethylenetriamine pentacetic acid (Gd DTPA) injection may serve to distinguish fluid from inflammation. We did not use Gd DTPA because the treatment of the patient would not have been influenced in the presence of a discharging fistula. Furthermore, Gd DTPA is expensive and an additional $\mathrm{T} 1$ sequence would prolong examination time.
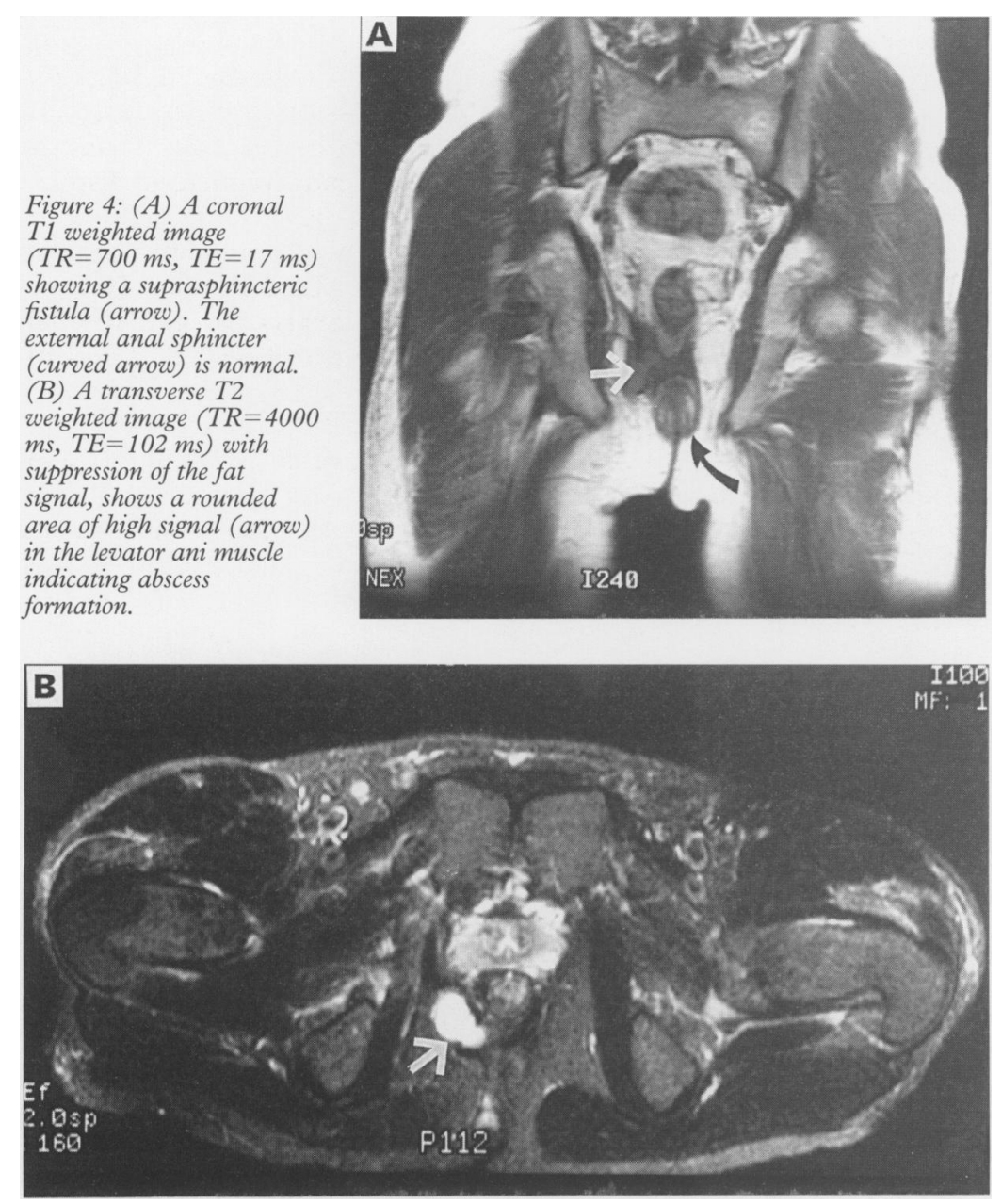

MRI was also successful in excluding fistula in ano. In no case was a fistula shown either clinically or at examination under anaesthetic when MRI was negative. MRI prospectively failed to detect one vesicoenteric fistula, although this was visible in retrospect.

The results of this study may be taken in context with previous studies. ${ }^{8-10}$ Lunniss et $a l^{8}$ used spin echo $\mathrm{T} 1$ and short Tau inversion recovery images at $0.5 \mathrm{~T}$ in the transverse and coronal planes. The short Tau inversion recovery sequence provides good suppression of fat and inflammatory tissue and gives a high signal because of long $\mathrm{T} 1$ and $\mathrm{T} 2$ relaxation times. Although successful in detecting active inflammation, the short Tau inversion recovery sequence entails long acquisition times and the images tend to be of poorer spatial resolution than equivalent spin echo sequences. This may have contributed to the difficulty in distinguishing the internal sphincter from the anal mucosa. ${ }^{8}$ Skalej et $a l^{9}$ also showed the value of MRI in the detection of perianal complications. They showed simple fistulas in 13 of 16 and complex fistulas in 18 of 18 patients who had Crohn's disease and suspected perianal disease. The sensitivity of $M R I$ is equivalent to the results that we report, however, their group is not fully representative and the outcome may be biased in that the specificity of MRI was not assessed.

Our examinations were obtained using the body coil and not a phased array multicoil system. Phased array multicoils would have the advantage of an improved signal to noise ratio, but would not influence spatial resolution for a fixed field of view. An endorectal coil combines the advantages of an increased signal to noise ratio and a small field of view; we do not have an endorectal coil. Although endorectal coils are used to stage prostatic carcinoma, ${ }^{11}$ there are no reported studies of perianal disease using an endorectal coil. The smaller field of view may be a disadvantage in the assessment of pelvic sidewall extension, and the distension of the $100 \mathrm{ml}$ balloon would be very painful in this group of patients.

The high sensitivity and specificity achieved in this study may be attributed to the use of multiplanar high resolution T2 images. This is only possible in a clinically manageable appointment time with the use of fast spin echo sequences. Fat suppression is a valuable adjunct because fat has a higher signal intensity on fast spin echo sequences than a comparable conventional spin echo sequence. ${ }^{12}$ Small foci of high signal in fistula tracks are appreciated more readily when fat suppression is used. Fat suppression by selective radiofrequency presaturation is easier to perform at $1.5 \mathrm{~T}$ because the resonant frequency of fat is $220 \mathrm{~Hz}$ higher than water. The efficiency of suppression may not be uniform, however, and care must be taken to place the patient close to the centre of the magnet to maximise homogeneity of fat suppression.

We conclude that MRI is a valuable technique in the assessment of patients with active perianal Crohn's disease. MRI offers a nonionising painless alternative to examination 
under anaesthetic and provides valuable information for preoperative planning in cases of complex fistulas and abscess formation.

1 The National Cooperative Crohn's Disease study. Gastroenterology 1979; 77: 829 .

2 Parks AG, Gordon PH, Hardcastle JD. A classification of fistula-in-ano. Br $f$ Surg 1976; 63: 1-12.

3 Park AG Morson BC. Proc Roy Soc Med 1962. 55: 751-4. 4 Choen S, Burnett S, Bartram CI, Nicholls RJ. Comparison between anal endosonography and digital examination in the evaluation of anal fistulae. $B r \mathcal{F}$ Surg 1991; 78: 445-7. 5 Kuijpers HC, Schulpen T. Fistulography for fistula-in-ano. Dis Colon Rectum 1985; 28: 103-4.

6 Yousem DM, Fishman EK, Jones B. Crohn's disease; perirectal and perianal findings at CT. Radiology 1988; 167: $331-4$.

7 Dosimetry Working Party of the Institute of Physical Sciences in Medicine. National Protocol for Patient Dose
Measurements in Diagnostic Radiology. 1992: National Radiological Protection Board.

8 Lunniss PJ, Armstrong P, Barker PG, Reznek RH, Phillips RKS. Magnetic resonance imaging of anal fistulae. Lancet 1992; 340: 394-6.

9 Skalej M, Bongers $\mathrm{H}$, Aicher H, Weinlich M, Starlinger M, Jenss $H$. Value of MR tomography in perianal Crohn's disease; a prospective study. Gastroenterology 1992; 102 (suppl); A697.

10 Koelbel G, Schmiedl U, Majer MC, Weber P, Jenss H, Kueper $\mathrm{K}$, et al. Diagnosis of fistulae and sinus tracts in patients with Crohn's disease: value of MR imaging. AfR 1989; 152: 999-1003.

11 Mirowitz AS, Brown JJ, Heiken JP. Evaluation of the prostate and prostatic carcinoma with gadolinium enhanced endorectal coil magnetic resonance imaging. Radiology 1993; 186: 153-7.

12 Hinks RS, Martin D. Bright fat, fast spin echo and CPMG Proceedings of the 11th Annual Scientific Meeting of the Society of Magnetic Resonance in Medicine, 1992: 4503. 